Article

\title{
PLA Melt Stabilization by High-Surface-Area Graphite and Carbon Black
}

\author{
Luciana D’Urso ${ }^{1}$, Maria Rosaria Acocella ${ }^{1}$ (D), Gaetano Guerra ${ }^{1}$, Valentina Iozzino ${ }^{2}$, \\ Felice De Santis $^{2}$ (iD) and Roberto Pantani ${ }^{2, *}$ (iD) \\ 1 Department of Chemistry and Biology, University of Salerno, Via Giovanni Paolo II 132, \\ 84084 Fisciano (SA), Italy; ldurso@unisa.it (L.D.); macocella@unisa.it (M.R.A.); gguerra@unisa.it (G.G.) \\ 2 Department of Industrial Engineering, University of Salerno, Via Giovanni Paolo II 132, \\ 84084 Fisciano (SA), Italy; viozzino@unisa.it (V.I.); fedesant@unisa.it (F.D.S.) \\ * Correspondence: rpantani@unisa.it; Tel.: +39-089-964-141
}

Received: 31 December 2017; Accepted: 30 January 2018; Published: 1 February 2018

\begin{abstract}
Small amounts of carbon nanofillers, specifically high-surface-area graphite (HSAG) and more effectively carbon black (CB), are able to solve the well-known problem of degradation (molecular weight reduction) during melt processing, for the most relevant biodegradable polymer, namely poly(lactic acid), PLA. This behavior is shown by rheological measurements (melt viscosity during extrusion experiments and time sweep-complex viscosity) combined with gel permeation chromatography (GPC) experiments. PLA's molecular weight, which is heavily reduced during melt extrusion of the neat polymer, can remain essentially unaltered by simple compounding with only $0.1 \mathrm{wt} \%$ of $\mathrm{CB}$. At temperatures close to polymer melting by compounding with graphitic fillers, the observed stabilization of PLA melt could be rationalized by scavenging traces of water, which reduces hydrolysis of polyester bonds. Thermogravimetric analyses (TGA) indicate that the same carbon fillers, on the contrary, slightly destabilize PLA toward decomposition reactions, leading to the loss of volatile byproducts, which occur at temperatures higher than $300^{\circ} \mathrm{C}$, i.e., far from melt processing conditions.
\end{abstract}

Keywords: poly(lactic acid); carbon black; graphite

\section{Introduction}

It is well known that melt processing of poly(lactic acid) (PLA) (typically conducted at temperatures close to $200^{\circ} \mathrm{C}$ ) generally leads to degradation, i.e., high molecular weight reduction [1-4], even in nitrogen atmospheres [2-5].

Many different approaches have been proposed to achieve PLA melt stabilization [6-14]. In particular, stabilization by compounding with commercial antioxidants and water scavengers $[6,7]$, or with chain extenders (i.e., molecules that reconnect polymer chains broken due to moisture at elevated temperatures), such as organic phosphites [8-10] or functional polysilsesquioxane microspheres [11], has been described. Additional thermal stabilization approaches involve polymer crosslinking by suitable agents [12-14] and polymer-end protection by acetyl groups [15].

Many reports show, on the basis of thermogravimetric analyses (TGA), that several nanofillers, such as clays and organoclays [16-21], silica [22,23], lignin [24], and silk [25] and cellulose [26] nanocrystals, stabilize PLA with respect to decomposition reactions, leading to loss of low-molecular-weight byproducts, which occur at temperatures higher than $300{ }^{\circ} \mathrm{C}$ (i.e., very far from melt processing conditions). In recent years, many papers have been published on PLA composites with graphite-based nanofillers [27-46] and carbon black [47-50]. These papers report significant improvements in PLA's physical (mainly electrical) properties with only a small amount of filler. 
Some of these papers report that some carbon fillers, like other nanofillers [16-26], can stabilize PLA with respect to decomposition reactions, leading to volatile byproducts at a temperature higher than $300{ }^{\circ} \mathrm{C}[27,29,32,34,43-46,48,49]$. For instance, the addition of exfoliated graphite increases of $5-15{ }^{\circ} \mathrm{C}$ decomposition temperatures corresponding to a $5 \%$ weight loss $\left(T_{\mathrm{d}, 5 \%}\right)[27,29,32]$.

Stabilization of the polymer toward decomposition reactions leading to volatile byproducts at high temperatures (for PLA for $\mathrm{T}>300^{\circ} \mathrm{C}$ ) does not assure maintenance of polymer molecular weight during melt processing (for PLA at $\mathrm{T} \approx 200^{\circ} \mathrm{C}$ ). In fact, some fillers (e.g., clays) that stabilize PLA toward high-temperature decomposition reactions destabilize PLA toward degradation reactions, leading to more pronounced polymer molecular weight reductions, in melt processing conditions [51-56]. In this respect, it is also worth adding that the temperatures corresponding to a $10 \%$ weight loss $\left(T_{\mathrm{d}, 10 \%}\right)$ for PLA and isotactic polypropylene (PP) were evaluated as $320^{\circ} \mathrm{C}$ and $270{ }^{\circ} \mathrm{C}$, while PP was incomparably more stable than PLA during melt processing [51].

In this paper, the influence of different graphite-based fillers-A low-surface-area graphite (LSAG), a high-surface-area graphite (HSAG), and a carbon black (CB) - On the stability of PLA melt at different temperatures is reported. In particular, PLA stability during melt processing (at $200{ }^{\circ} \mathrm{C}$ ) was studied via melt viscosity measurements during extrusion, via evaluations of molecular weight distributions of the extruded unfilled and filled samples with gel permeation chromatography (GPC), and via rheological measurements (time sweep-complex viscosity). PLA stability at higher temperatures (above $300^{\circ} \mathrm{C}$ ), where decomposition reactions lead to loss of volatile byproducts, was studied via TGA.

It was found that the considered graphite-based fillers do not improve (or even slightly reduce) PLA thermal stability above $300{ }^{\circ} \mathrm{C}$, i.e., they marginally affect high temperature decomposition reactions, leading to low-molecular-mass byproducts. However, very small amounts (as low as $0.1 \mathrm{wt} \%$ ) of high-surface-area graphite and of carbon black, are sufficient to inhibit degradation reactions, leading to molecular weight reductions without a loss of volatile byproducts, which occur during processing at $200{ }^{\circ} \mathrm{C}$.

\section{Experimental}

In this work, a commercial grade of PLA produced by NatureWorks (Minnetonka, MN, USA) with the trade name of 4032D was adopted. This PLA grade has a D-enantiomer content of approximately $2 \%$ and a maximum degree of crystallinity of about $45 \%$. A thermal and rheological characterization of the material can be found in the literature [57-60].

The material was dried at $60{ }^{\circ} \mathrm{C}$ under vacuum overnight before any processing and testing operation.

Primary Synthetic Graphite TIMREX ${ }^{\circledR}$ SFG6 with a low surface area (LSAG, of about $17 \mathrm{~m}^{2} / \mathrm{g}$ ), an average particle size of $6 \mu \mathrm{m}$, and a carbon amount of $99.6 \%$ was provided by Timcal Graphite \& Carbon (Bodio, Switzerland). Synthetic Graphite TC 307 with a high surface area (HSAG, of about $352 \mathrm{~m}^{2} / \mathrm{g}$ ), a primary particle size less than $1 \mu \mathrm{m}$, a carbon amount of $99.92 \%$, and a high shape anisotropy of the crystallites [61] was purchased from Asbury Graphite Mills, Inc (Asbury, NJ, USA). The used carbon black sample (CB) of grade N660, with a surface area of $33 \mathrm{~m}^{2} / \mathrm{g}$ and a particle size around 49-60 nm, was purchased from Cabot Company (Boston, MA, USA).

Wide-angle X-ray diffraction (WAXD) patterns were obtained by an automatic Bruker D8 Advance diffractometer (Bruker Corp, Billerica, MA, USA), in reflection, at $35 \mathrm{kV}$ and $40 \mathrm{~mA}$, using nickel-filtered $\mathrm{Cu} \mathrm{K} \alpha$ radiation $(0.15418 \mathrm{~nm})$. The $d$ spacings were calculated using Bragg's law, and the observed integral breadths $\left(\beta_{\mathrm{obs}}\right)$ were determined by a fit with a Lorentzian function of the diffraction patterns. The instrumental broadening $\left(\beta_{\text {inst }}\right)$ was also determined by fitting of a Lorentzian function to line profiles of a standard silicon powder 325 mesh (99\%). The corrected integral breadths of the 002 peak were determined by subtracting the instrumental broadening of the closest silicon reflection from 
the observed integral breadths, $\beta=\beta_{\text {obs }}-\beta_{\text {inst }}$. The correlation lengths $(D)$ were determined using Scherrer's equation:

$$
D=\frac{K \lambda}{\beta \cos \theta}
$$

where $\lambda$ is the wavelength of the incident $X$-rays and $\theta$ the diffraction angle, assuming the Scherrer constant $K=1$.

Melt compounding was carried out by a microcompounder (twin screws, counter-rotating, Haake Mini-lab II, Thermo Fisher Scientific, Schwerte, Germany). Thanks to a backflow channel and a bypass valve, it is possible to define the residence time in the microcompounder. Furthermore, this device allows one to estimate the viscosity as the material is compounded in the back flow channel that is a rectangular slit with two pressure transducers $[62,63]$. The tests were conducted at a temperature of $200{ }^{\circ} \mathrm{C}$ and a screw rotation speed of $100 \mathrm{rpm}$. Under these conditions, the estimated shear rate at which the viscosity was calculated is about $350 \mathrm{~s}^{-1}$. After $15 \mathrm{~min}$, the bypass valve is opened and the compound is extruded.

The zero-shear rate viscosity of a polymer can be related to the molecular weight of the polymer by the following equation:

$$
\eta=c M w^{a}
$$

where $a$ is an exponent whose value is generally accepted to be 3.4 [64], and $c$ is a parameter that depends on temperature. According to Equation (2), due to the exponent $a$, the viscosity is extremely sensitive to changes in molecular weight, so rheological measurements are an extremely powerful means of assessing the degradation in the molten state.

The melt compounding was carried out for $15 \mathrm{~min}(900 \mathrm{~s})$ at $200{ }^{\circ} \mathrm{C}$. The materials were then taken from the microcompounder and used for the subsequent analysis: GPC and rheology.

GPC measurements were conducted by a Waters Breeze GPC system (Waters, Milford, MA, USA), equipped with a refractive index (RI) detector, by using a set consisting of four Styragel HT columns with $(102,103,104$, and $105 \AA$ pore size) and $10 \mu \mathrm{m}$ (particle size). Tetrahydrofuran, THF, was used as eluent at $35{ }^{\circ} \mathrm{C}$ at a flow rate of $1.0 \mathrm{~mL} \cdot \mathrm{min}^{-1}$. The calibration curve was established with polystyrene standards.

Time sweep experiments were performed by means of a Haake Mars II (Thermo Scientific) rotational rheometer in a plate-plate configuration $(D=20 \mathrm{~mm})$ under a dry nitrogen atmosphere. A constant stress of $100 \mathrm{~Pa}$ and a frequency of $1 \mathrm{rad} / \mathrm{s}$ were applied during the tests. In this condition, all measurements were carried out within the linear response domain and within the Newtonian plateau for all materials. The tests were carried out at a temperature of $200{ }^{\circ} \mathrm{C}$ for about $3 \mathrm{~h}$.

TGA analyses were conducted with a TG 209 F1, manufactured by Netzsch Geraetebau (Selb, Germany), with a heating rate of $10 \mathrm{~K} / \mathrm{min}$ under an $\mathrm{N}_{2}$ flow.

DSC scans were conducted at a heating rate of $10 \mathrm{~K} / \mathrm{min}$. and the results are reported in the supplementary material (Figure S1).

\section{Results and Discussion}

\subsection{WAXD Characterization of Carbon Fillers}

WAXD patterns, as collected by an automatic powder diffractometer, of the used low-surface-area (blue curve) and high-surface-area (red curve) graphites are compared in Figure 1. It is immediately apparent that their crystalline structures are largely different. In particular, LSAG exhibits a much more ordered structure, with a large number of narrow diffraction peaks. These peaks can be easily indexed by assuming the presence of both hexagonal or rhombohedral phases $[65,66]$. Particularly informative is the pattern region with $42^{\circ}<2 \theta_{\mathrm{Cu} \mathrm{K} \alpha}<46^{\circ}$, where four well defined peaks are present, with two peaks at $d=0.213 \mathrm{~nm}$ and $d=0.205 \mathrm{~nm}$ indexed as (100) and (101) reflections of the hexagonal phase and two peaks at $d=0.209 \mathrm{~nm}$ and $d=0.197 \mathrm{~nm}$ indexed as (101) and (102) reflections of the 
rhombohedral phase. The fraction of rhombohedral modification is approximately $30 \%$, as derived by comparing the integrated intensities for the above cited hexagonal and rhombohedral peaks.
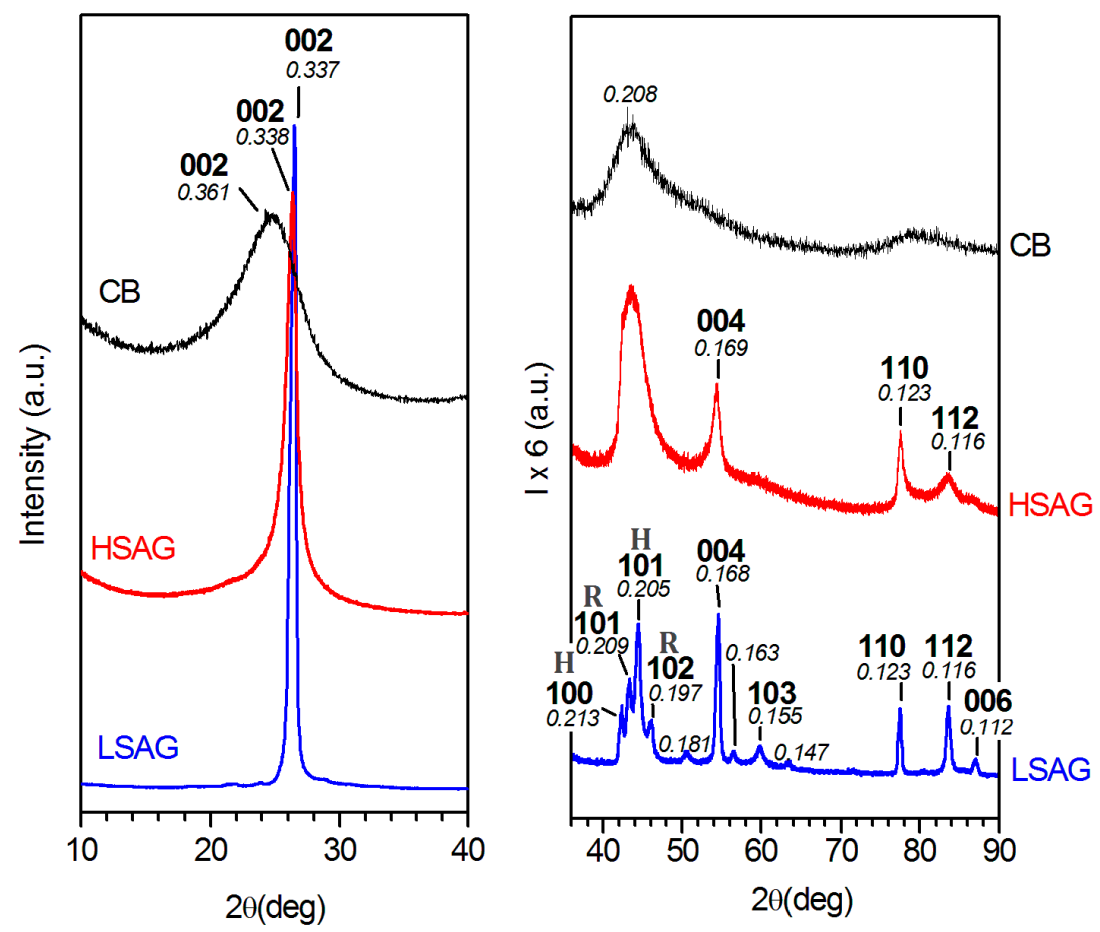

Figure 1. WAXD patterns $(\mathrm{Cu} \mathrm{K} \alpha)$, as collected by an automatic powder diffractometer, of used carbon fillers: LSAG (lower blue curve); HSAG (intermediate red curve); CB (upper black curve). $\mathrm{H}$ and $\mathrm{R}$ labels refer to reflections being specific of hexagonal and rhombohedral phases, respectively.

HSAG exhibits a much more disordered structure, with a strongly reduced number of diffraction peaks. In particular, besides (001) reflections and the in-plane 110 reflection at $d=0.123 \mathrm{~nm}$, only a broad diffraction halo is present that is roughly centered at $d=0.208 \mathrm{~nm}$. This clearly indicates the occurrence of a turbostratic graphite with a nearly complete disorder in the relative position of parallel graphitic layers $[61,65,67]$.

For both LSAG and HSAG, the distance between parallel graphitic layers is equal to $d=0.337 \mathrm{~nm}$, while the corresponding correlation length perpendicular to the graphitic planes (as evaluated by breadths of the 002 peak) is much lower for HSAG, with $D_{\perp, \mathrm{HSAG}}=12 \mathrm{~nm}$ and $D_{\perp, \mathrm{LSAG}}=26 \mathrm{~nm}$.

For the sake of comparison, the WAXD pattern of the used carbon black is also shown in Figure 1. As discussed in detail in a recent paper [68], WAXD patterns of CB (as well as of oxidized CB, oCB) samples suggest that they are prevailingly constituted by a disordered spatial arrangement of highly defective structural layers with short in-plane correlation lengths $(2-3 \mathrm{~nm})$. This was confirmed by the ability of oCB to form ordered intercalation compounds [68].

\subsection{Melt Compounding of PLA in the Presence of Different Kinds and Amounts of Carbon Fillers}

Viscosity values measured during compounding are reported in Figure 2. The data are normalized with respect to the initial values measured for each material, so that all values start from 1 . The curve which refers to the neat PLA is reported for comparison in all the plots of Figure 2. It can be seen that, in agreement with that reported in the literature [5,69], the viscosity of pure PLA during compounding immediately starts to reduce significantly, such that viscosity becomes about one half of the starting value after about $15 \mathrm{~min}$. According to Equation (2), this reduction suggests a reduction of about $20 \%$ in the molecular weight of the material. 
All the fillers adopted, with the exception of graphite LSAG at the lowest used percentage of $0.1 \%$, introduce a significant stabilizing effect, such that the reduction of viscosity with time is limited to about $10 \%$. For graphite HSAG and CB, this effect is reached already for filler contents of $0.1 \%$, which is an extremely significant result for PLA processing.

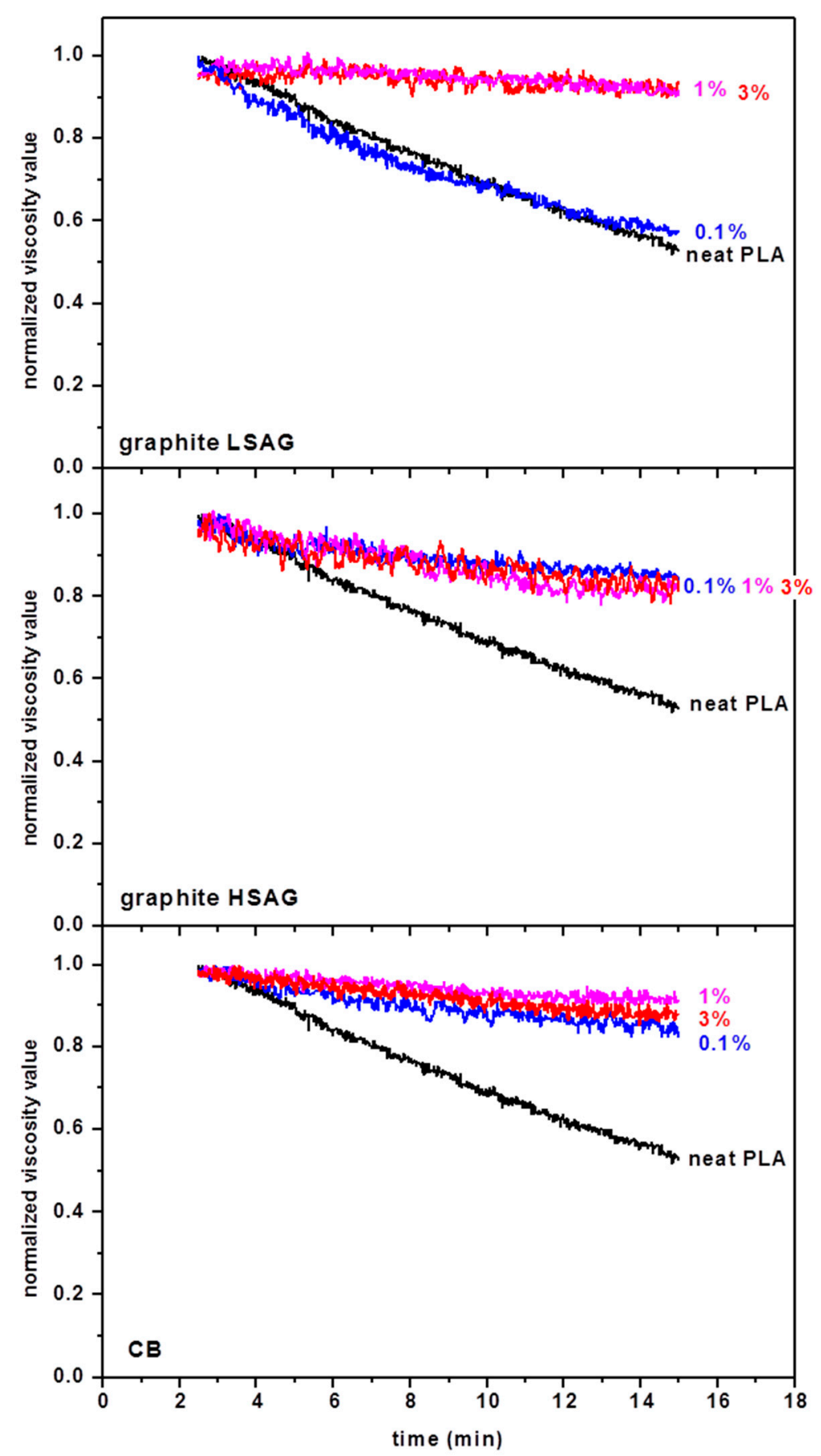

Figure 2. Time evolution of normalized viscosity (with respect to the initial value) in the microcompounder at $T=200{ }^{\circ} \mathrm{C}$ and $100 \mathrm{rpm}$. The estimated value of shear rate is about $350 \mathrm{~s}^{-1}$.

GPC curves of PLA pellet and of extruded PLA compounds, with different kinds and amounts of carbon fillers, are shown in Figure 3. 
Elution times of GPC curves of PLA are definitely lower before extrusion (green lowest curve in Figure 3) than after extrusion (black curve in Figure 3). This confirms that, as generally observed for PLA, extrusion processes lead to a substantial polymer degradation. As shown in Table 1, GPC curves indicate a reduction of about $25 \%$ of the initial number-average molecular weights ( $M n$ and $M w$ ). This is consistent with a reduction in viscosity of about $40 \%$, in agreement with the results shown in Figure 2.

GPC curves of the extruded PLA compounds indicate that, for all the considered carbon fillers, a concentration of $3 \mathrm{wt} \%$ (continuous lines in Figure 3) is able to eliminate the adverse effect of the considered PLA processing on molecular mass. Moreover, for HSAG and CB, a concentration as low as $0.1 \mathrm{wt} \%$ is sufficient to stabilize PLA to the molecular mass of the virgin pellet (dotted curves in Figure 3, 7th and 10th columns in Table 1). Again, these results are consistent with rheological data reported in Figure 2.

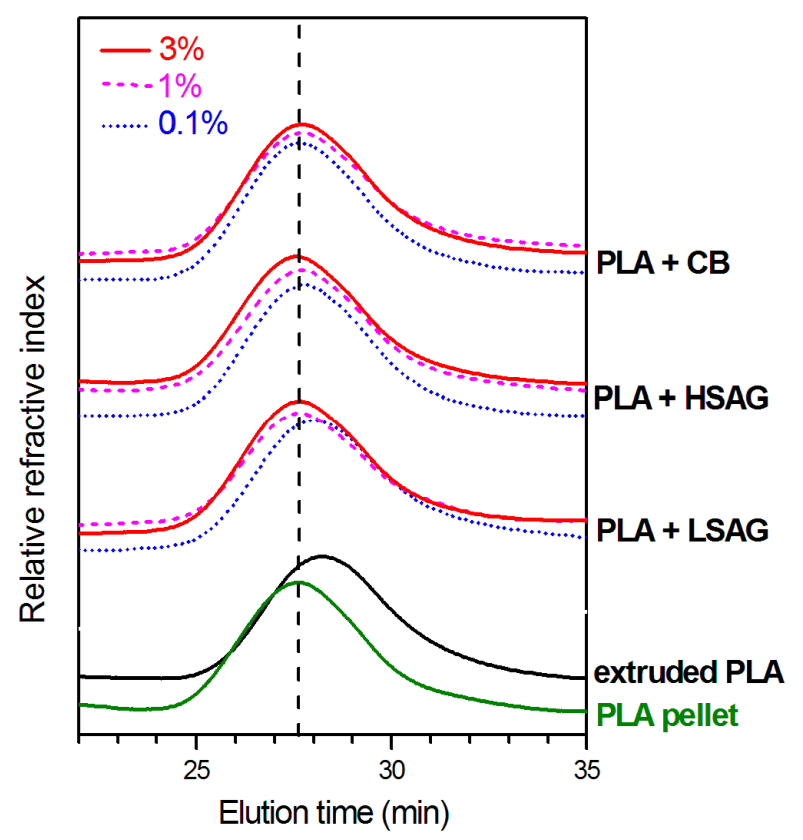

Figure 3. GPC curves in THF at $35{ }^{\circ} \mathrm{C}$ of the PLA pellet (green) of extruded PLA (black) as well as of extruded PLA compounds, as obtained by processes whose viscosity reduction is shown in Figure 2. Compounds contain $0.1 \mathrm{wt} \%$ (dotted lines), $1 \mathrm{wt} \%$ (dashed lines), and $3 \mathrm{wt} \%$ (continuous lines) of different graphitic fillers: LSAG, HSAG, and CB.

Table 1. Number average molecular weight $(M n)$, weight average molecular weight $(M w)$, and polydispersity index (PDI) as evaluated by GPC curves, for PLA pellet and extruded compounds with LSAG, HSAG, and $\mathrm{CB}$. The evaluated variance is of $\pm 3 \mathrm{kDa}$.

\begin{tabular}{|c|c|c|c|c|c|c|c|c|c|c|c|}
\hline & \multicolumn{11}{|c|}{ Extruded PLA Samples } \\
\hline & \multirow{2}{*}{ PLA Pellet } & \multirow{2}{*}{ PLA Neat } & \multicolumn{3}{|c|}{ LSAG } & \multicolumn{3}{|c|}{ HSAG } & \multicolumn{3}{|c|}{ СВ } \\
\hline & & & $0.1 \%$ & $1 \%$ & $3 \%$ & $0.1 \%$ & $1 \%$ & $3 \%$ & $0.1 \%$ & $1 \%$ & $3 \%$ \\
\hline$M n[\mathrm{kDa}]$ & $120 \pm 3$ & 88 & 95 & 120 & 120 & 120 & 121 & 120 & 121 & 121 & 120 \\
\hline$M w[\mathrm{kDa}]$ & $195 \pm 3$ & 146 & 165 & 195 & 196 & 190 & 194 & 196 & 192 & 193 & 193 \\
\hline
\end{tabular}

The PLA pellet and the extruded PLA compounds were analyzed by time sweep rheological tests. The results are reported in Figure 4 and show even more clearly the thermal stabilization effect of carbon fillers. These measurements, which were carried out for a very long time (about $3 \mathrm{~h}$ ) indicate a degradation at high temperature after the microcompounding step. Under these conditions, it is possible to discriminate between stabilization effects of HSAG and CB, which would appear to be very 
similar on the basis of the viscosity measurements of Figure 2 as well as on the basis of the GPC data of Figure 3 and Table 1, which indicate the effect of the microcompounding step.

The viscosity evolution reported in Figure 4 can be interpreted in terms of molecular weight according to Equation (2). The results are reported in Figure 5.

The initial molecular weight, $M w(t=0)$, indicates the value after the microcompounding step for all the samples except the PLA in pellet. The stabilizing effect of the fillers is clearly evidenced: the virgin polymer (both neat and extruded) presents a reduction of about $30 \%$ in molecular weight during the $3 \mathrm{~h}$ of the test at $200{ }^{\circ} \mathrm{C}$. All considered carbon fillers indicate a slower decrease in $\mathrm{Mw}$, with an effect that generally depends on concentration: an increase from $0.1 \%$ to $1 \%$ induces a slower degradation. For $\mathrm{CB}, 0.1 \%$ and $1 \%$ induce the same effect and allow for a reduction of just $10 \%$ of $\mathrm{Mw}$ during the test.

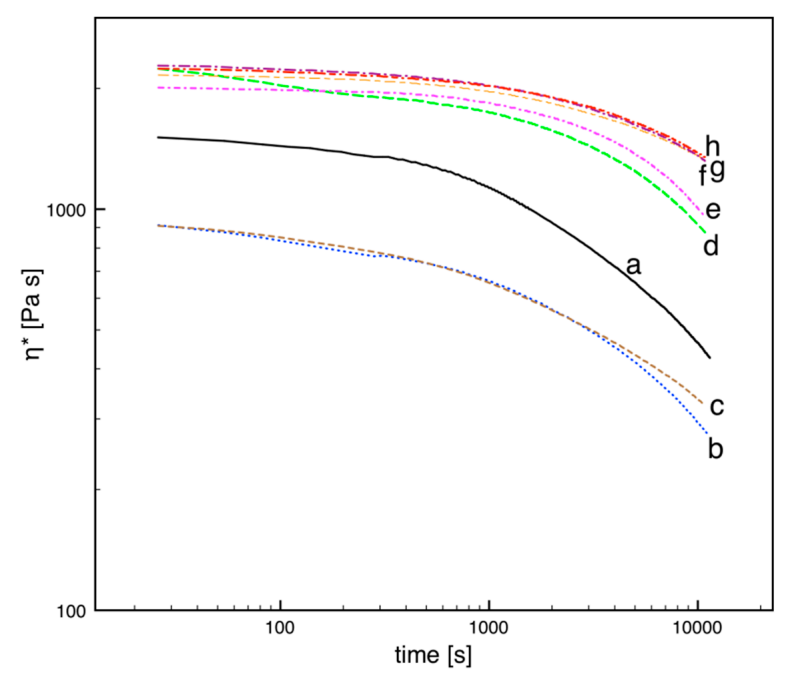

Figure 4. Time sweep-complex viscosity for the virgin pellet (a) and for extruded PLA samples after microcompounding: (b) neat and with (c) $0.1 \mathrm{wt} \%$ of LSAG; (d) $1 \mathrm{wt} \%$ of LSAG; (e) $0.1 \mathrm{wt} \%$ of HSAG; (f) $1 \mathrm{wt} \%$ of HSAG; (g) $0.1 \mathrm{wt} \%$ of CB; (h) $1 \mathrm{wt} \%$ of CB. Experimental conditions: $T=200{ }^{\circ} \mathrm{C}$, $f=1 \mathrm{rad} / \mathrm{s}$, plate-plate, gap $=200 \mu \mathrm{m}$.

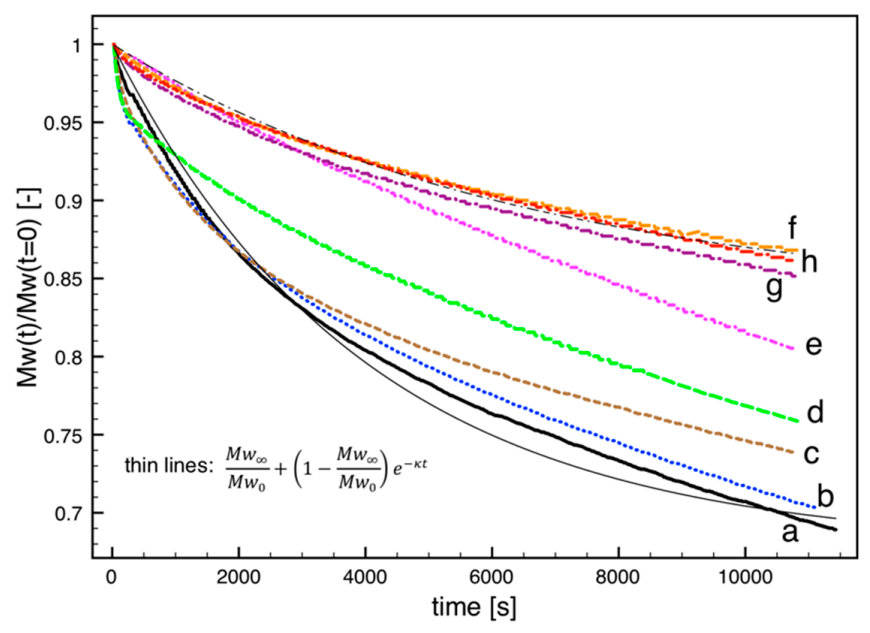

Figure 5. Time sweep-complex viscosity for the virgin PLA pellet (a) and for extruded samples after microcompounding: (b) neat and with (c) $0.1 \mathrm{wt} \%$ of LSAG; (d) $1 \mathrm{wt} \%$ of LSAG; (e) $0.1 \mathrm{wt} \%$ of HSAG; (f) 1 wt \% of HSAG; (g) $0.1 \mathrm{wt} \%$ of CB; (h) $1 \mathrm{wt} \%$ of CB. Experimental conditions: $T=200{ }^{\circ} \mathrm{C}$, $f=1 \mathrm{rad} / \mathrm{s}$, plate-plate, gap $=200 \mu \mathrm{m}$. 
The obtained results appear to be nearly independent of the surface area of carbon black. In fact, similar PLA melt stabilization was obtained using a different carbon black sample, exhibiting a definitely higher surface area (N110 with surface area of $150 \mathrm{~m}^{2} / \mathrm{g}$ ).

The observed polyester melt stabilization by HSAG and mainly by carbon black could be explained by the removal of traces of water from the melt, thus strongly reducing the hydrolysis of polyester bonds.

Indeed, on assuming that water is the only reason for molecular chain scission, namely assuming that hydrolysis is the only degradation mechanism (which is surely a simplification of more complex mechanisms taking place at high temperatures), one can relate the amount of water to the amount of carboxylic end groups according to the following equations:

$$
\frac{d C a}{d t}=-\frac{d C c}{d t} \Rightarrow C a=\left(C c_{0}+C a_{0}\right)-C c=C c_{\infty}-C c
$$

in which $C a$ is the concentration of water inside the samples and $C c$ is the concentration of carboxylic end groups. The subscripts 0 and $\infty$ indicate the initial concentrations (at $t=0$ ) and the final situation in which water molecules completely disappeared, respectively.

Considering that the concentration of carboxylic end groups is related to the concentration of polymeric chains

$$
C_{c}=\frac{\rho}{M n}
$$

one simply obtains

$$
C a=\frac{\rho}{M n_{\infty}}\left(1-\frac{M n_{\infty}}{M n}\right)
$$

and finally

$$
C a_{0}=\frac{\rho}{M n_{\infty}}\left(1-\frac{M n_{\infty}}{M n_{0}}\right)
$$

Assuming that, at least during early stages of degradation, the polydispersity index ( $\mathrm{Mw} / \mathrm{Mn}) \mathrm{can}$ be considered to be constant, as also confirmed by the GPC data reported in Table 1, the data reported in Figure 5 can be considered to refer to $\mathrm{Mn} / \mathrm{Mn}_{0}$ during melt degradation. Those time evolutions can be fitted by a simple exponential curve, which can provide an estimate of the molecular weight at long times. For the samples a and $\mathrm{h}$ in Figure 5, the exponential fitting is reported. On knowing the density $\left(1.2 \mathrm{~g} / \mathrm{cm}^{3}\right)$ and the initial molecular weight from GPC data reported in Table 1, the initial water concentration is easily calculated. For the sample a (neat, virgin material), $C a_{0}$ is $4.7 \mathrm{~mol} / \mathrm{m}^{3}$, corresponding to about $70 \mathrm{ppm}$. For the sample $\mathrm{h}(1 \mathrm{wt} \%$ of $\mathrm{CB}), \mathrm{C} a_{0}$ is $2 \mathrm{~mol} / \mathrm{m}^{3}$, corresponding to about $30 \mathrm{ppm}$. The indication provided by the simplified model described above is that the amount of water taking part in the hydrolysis can be substantially reduced (of a factor 2 or more) by adding the fillers analyzed in this work.

This water absorption possibly occurs by specific interactions between water and oxidized groups on carbon surfaces [70].

\subsection{Weight Loss in TGA Experiments of PLA in the Presence of Carbon Fillers}

TGA scans of the extruded compounds indicate that PLA thermal stability, expressed in terms of weight loss, is slightly reduced by compounding with all the considered carbon fillers. Just as an example, TGA scans with a heating rate of $10 \mathrm{~K} / \mathrm{min}$ for the PLA pellet, for the neat PLA-extruded sample, and for the extruded nanocomposites with $0.1 \mathrm{wt} \%$ of filler concentration are compared in Figure 6.

An increase in the decomposition temperature, e.g., corresponding to a $5 \%$ of weight loss $\left(T_{\mathrm{D}, 5 \%}\right)$, is observed going from the virgin $\left(T_{\mathrm{D}, 5 \%}=327^{\circ} \mathrm{C}\right)$ to the extruded neat $\left(T_{\mathrm{D}, 5 \%}=332{ }^{\circ} \mathrm{C}\right)$ sample, although the latter has undergone a remarkable molecular weight reduction (GPC data of Table 1). 
Moreover, compounding with all considered carbon fillers leads to small decreases of $T_{\mathrm{D}, 5 \%}$, mainly for $\mathrm{CB}\left(T_{\mathrm{D}, 5 \%}=328^{\circ} \mathrm{C}\right)$.

The results of Section 3.2 clearly indicate that carbon fillers stabilize PLA toward degradation reactions occurring at temperatures close to $200{ }^{\circ} \mathrm{C}$, not far from PLA's melting temperature, which leads to a reduction in the molecular masses, without any loss of volatile degradation products. The TGA results of this section indicate that the same carbon fillers, on the contrary, slightly destabilize PLA toward decomposition reactions occurring at temperatures higher than $300{ }^{\circ} \mathrm{C}$, which leads to a loss of volatile byproducts.

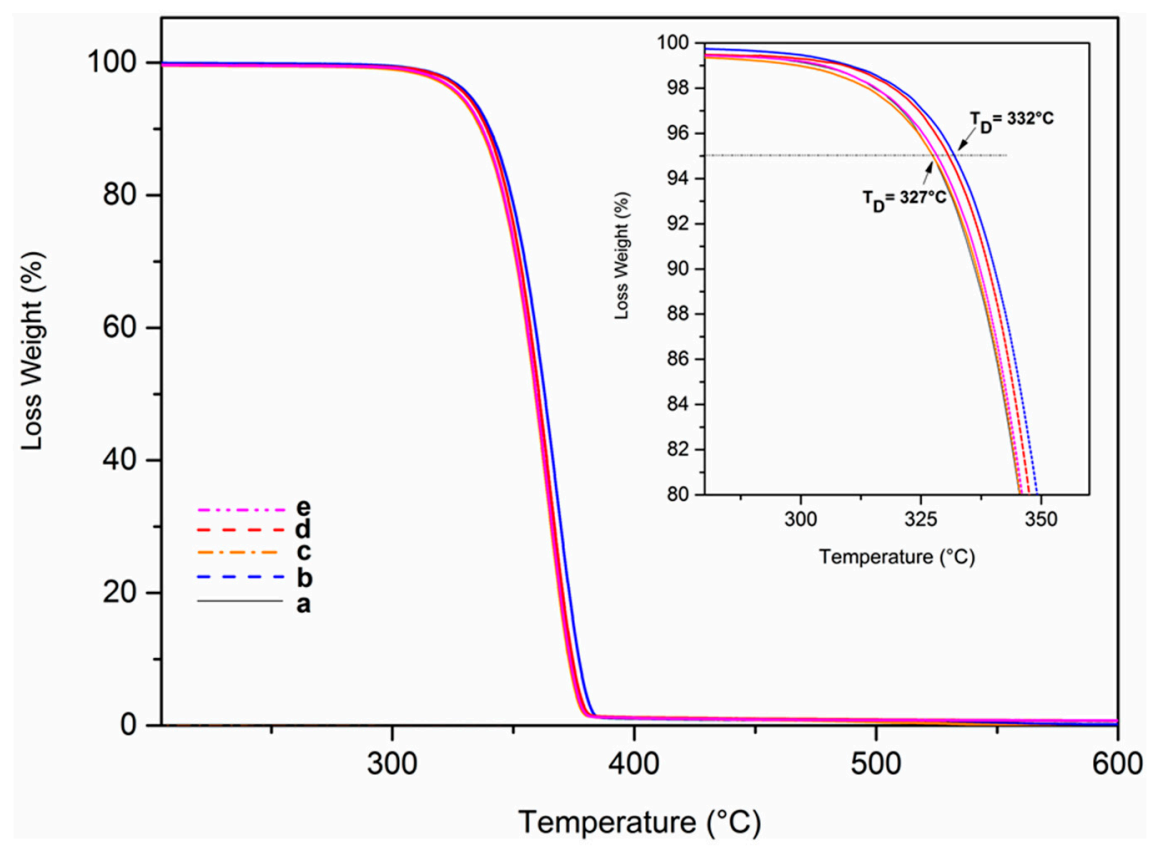

Figure 6. TGA scans for the virgin PLA pellet (a) and for extruded samples after microcompounding: (b) neat and with (c) 0.1 wt \% of LSAG; (d) 0.1 wt \% of HSAG; (e) 0.1 wt \% of CB. Experimental conditions: a heating rate of $10 \mathrm{~K} / \mathrm{min}$ under an $\mathrm{N}_{2}$ atmosphere.

\section{Conclusions}

Different carbon fillers, specifically low- and high-surface-area graphite as well as carbon black, have been tested as possible stabilizers of PLA melt.

Melt viscosity measurements during extrusion processes and GPC experiments on the corresponding extruded samples show a remarkable PLA melt stabilization by all the considered nanofillers, at a temperature just above melting. In particular, melt stabilization leads to degradation reactions, leading to molecular weight reduction without any weight loss. For instance, for extrusions conducted at $200{ }^{\circ} \mathrm{C}$, neat PLA exhibits a molecular weight reduction of about $25 \%$ while, by compounding with only $0.1 \mathrm{wt} \%$ of HSAG or of CB, PLA's molecular weight remains unaltered.

Time sweep-complex viscosity measurements, as carried out at $200^{\circ} \mathrm{C}$ for about $3 \mathrm{~h}$, confirm the ability of carbon nanofillers to stabilize PLA toward degradation reactions. In fact, PLA's molecular weight was reduced by about $30 \%$ in the virgin polymer (both neat and extruded), while it was reduced by amounts in the range $25-10 \%$ in the considered carbon composites. These measurements show that CB most effectively slowed down PLA degradation, for which a content of only $0.1 \mathrm{wt} \%$ leads to a molecular weight reduction close to $10 \%$, much lower than that of the neat PLA (30\%).

TGA analyses indicate that the considered carbon fillers, on the contrary, slightly destabilize PLA toward decomposition reactions, leading to a loss of volatile byproducts, which occur at temperatures higher than $300^{\circ} \mathrm{C}$, i.e., far from melt processing conditions. 
The observed PLA stabilization by carbon fillers, at temperatures suitable for melt processing, can be explained by scavenging traces of water from the melt, which reduces the hydrolysis of polyester bonds.

In summary, PLA compounding with very small amounts (even $0.1 \mathrm{wt} \%$ ) of HSAG and CB lead to remarkable PLA stabilization toward reactions, leading to molecular weight reduction. This contributes to the solution of the well-known problem of PLA degradation during processing. The same carbon nanofillers can also be effective in reducing degradation during processing for other polyesters that are sensitive to hydrolysis in the melt state.

Supplementary Materials: The following are available online at http:/ /www.mdpi.com/2073-4360/10/2/139/s1, Figure S1: DSC heating scans of the extruded PLA and PLA compounded with $0.1 \mathrm{wt} \%$ of HSAG, LSAG and CB.

Acknowledgments: We thank Mario Maggio of University of Salerno for useful discussions. Financial support of "Ministero dell' Istruzione, dell'Università e della Ricerca" is gratefully acknowledged.

Author Contributions: Gaetano Guerra and Roberto Pantani conceived and designed the experiments; Luciana D'Urso and Maria Rosaria Acocella prepared the fillers; Valentina Iozzino prepared the compounds; Luciana D'Urso and Valentina Iozzino performed the experiments; Maria Rosaria Acocella and Felice De Santis analyzed the data; Luciana D'Urso wrote the paper.

Conflicts of Interest: The authors declare no conflict of interest.

\section{References}

1. Degee, P.; Dubois, P.; Jerome, R. Bulk polymerization of lactides initiated by aluminium isopropoxide, 3. Thermal stability and viscoelastic properties. Macromol. Chem. Phys. 1997, 198, 1985-1995. [CrossRef]

2. Wachsen, O.; Platkowski, K.; Reichert, K.H. Thermal degradation of poly-L-lactide-studies on kinetics, modelling and melt stabilisation. Polym. Degrad. Stab. 1997, 57, 87-94. [CrossRef]

3. Signori, F.; Coltelli, M.B.; Bronco, S. Thermal degradation of poly(lactic acid) (PLA) and poly(butylene adipate-co-terephthalate) (PBAT) and their blends upon melt processing. Polym. Degrad. Stab. 2009, 94, 74-82. [CrossRef]

4. Carrasco, F.; Pagès, P.; Gámez-Pérez, J.; Santana, O.O.; Maspoch, M.L. Processing of poly(lactic acid): Characterization of chemical structure, thermal stability and mechanical properties. Polym. Degrad. Stab. 2010, 95, 116-125. [CrossRef]

5. Speranza, V.; De Meo, A.; Pantani, R. Thermal and hydrolytic degradation kinetics of PLA in the molten state. Polym. Degrad. Stab. 2014, 100, 37-41. [CrossRef]

6. Gruber, P.R.; Kolstad, J.J.; Ryan, C.M.; Hall, E.S.; Eichen, C.R.S. Melt-Stable Amorphous Lactide Polymer Film and Process for Manufacturing Thereof. U.S. Patent 5,484,881 A, 16 January 1996.

7. Amorin, N.S.Q.S.; Rosa, G.; Fernandes Alves, J.; Goncalves, S.P.C.; Franchetti, S.M.M.; Fechine, G.J.M. Study of thermodegradation and thermostabilization of poly(lactide acid) using subsequent extrusion cycles. J. Appl. Polym. Sci. 2014, 131, 40023. [CrossRef]

8. Cheung, M.F.; Carduner, K.R.; Golovoy, A.; Van Oene, H. Studies on the role of organophosphites in polyester blends: II. The inhibition of ester-exchange reactions. J. Appl. Polym. Sci. 1990, 40, 977-987. [CrossRef]

9. Cicero, J.A.; Dorgan, J.R.; Dec, S.F.; Knauss, D.M. Phosphite stabilization effects on two-step melt-spun fibers of polylactide. Polym. Degrad. Stab. 2002, 78, 95-105. [CrossRef]

10. Meng, X.; Shi, G.; Wu, C.; Chen, W.; Xin, Z.; Shi, Y.; Sheng, Y. Chain extension and oxidation stabilization of Triphenyl Phosphite (TPP) in PLA. Polym. Degrad. Stab. 2016, 124, 112-118. [CrossRef]

11. Han, T.; Xin, Z.; Shi, Y.; Zhao, S.; Meng, X.; Xu, H.; Zhou, S. Control of thermal degradation of poly(lactic acid) using functional polysilsesquioxane microspheres as chain extenders. J. Appl. Polym. Sci. 2015, 132, 41977. [CrossRef]

12. Yang, S.; Wu, Z.H.; Yang, W.; Yang, M.B. Thermal and mechanical properties of chemical crosslinked polylactide (PLA). Polym. Test. 2008, 27, 957-963. [CrossRef]

13. Yang, L.; Chen, X.; Jing, X. Stabilization of poly(lactic acid) by polycarbodiimide. Polym. Degrad. Stab. 2008, 93, 1923-1929. [CrossRef] 
14. Bai, H.; Liu, H.; Bai, D.; Zhang, Q.; Wang, K.; Deng, H.; Chen, F.; Fu, Q. Enhancing the melt stability of polylactide stereocomplexes using a solid-state cross-linking strategy during a melt-blending process. Polym. Chem. 2014, 5, 5985-5993. [CrossRef]

15. Fan, Y.; Nishida, H.; Shirai, Y.; Endo, T. Thermal stability of poly(L-lactide): Influence of end protection by acetyl group. Polym. Degrad. Stab. 2004, 84, 143-149. [CrossRef]

16. Pluta, M.; Galeski, A.; Alexandre, M.; Paul, M.-A.; Dubois, P. Polylactide/montmorillonite nanocomposites and microcomposites prepared by melt blending: Structure and some physical properties. J. Appl. Polym. Sci. 2002, 86, 1497-1506. [CrossRef]

17. Paul, M.-A.; Alexandre, M.; Degee, P.; Henrist, C.; Rulmont, A.; Dubois, P. New nanocomposite materials based on plasticized poly(L-lactide) and organo-modified montmorillonites: Thermal and morphological study. Polymer 2003, 44, 443-450. [CrossRef]

18. Pluta, M. Melt compounding of polylactide/organoclay: Structure and properties of nanocomposites. J. Polym. Sci. B 2006, 44, 3392-3405. [CrossRef]

19. Wu, T.-M.; Wu, C.-Y. Biodegradable poly(lactic acid)/chitosan-modified montmorillonite nanocomposites: Preparation and characterization. Polym. Degrad. Stab. 2006, 91, 2198-2204. [CrossRef]

20. Iturrondobeitia, M.; Okariz, A.; Guraya, T.; Zaldua, A.M.; Ibarretxe, J. Influence of the processing parameters and composition on the thermal stability of PLA/nanoclay bio-nanocomposites. J. Appl. Polym. Sci. 2014, 131, 40747. [CrossRef]

21. Krishnaiah, P.; Ratnam, C.T.; Manickam, S. Development of silane grafted halloysite nanotube reinforced polylactide nanocomposites for the enhancement of mechanical, thermal and dynamic-mechanical properties. Appl. Clay Sci. 2017, 135, 583-595. [CrossRef]

22. Huang, J.-W.; Hung, Y.C.; Wen, Y.-L.; Kang, C.-C.; Yeh, M.-Y. Polylactide/nano and microscale silica composite films. I. Preparation and characterization. J. Appl. Polym. Sci. 2009, 112, 1688-1694. [CrossRef]

23. Wen, X.; Zhang, K.; Wang, Y.; Han, L.; Han, C.; Zhang, H.; Chen, S.; Dong, L. Study of the thermal stabilization mechanism of biodegradable poly(L-lactide)/silica nanocomposites. Polym. Int. 2011, 60, 202-210. [CrossRef]

24. Gordobi, O.; Delucis, R.; Egues, I.; Labidi, J. Kraft lignin as filler in PLA to improve ductility and thermal properties. Ind. Crops Prod. 2015, 72, 46-53. [CrossRef]

25. Tesfaye, M.; Patwa, R.; Gupta, A.; Kashyap, M.J.; Katiyar, V. Recycling of poly(lactic acid)/silk based bionanocomposites films and its influence on thermal stability, crystallization kinetics, solution and melt rheology. Int. J. Biol. Macromol. 2017, 101, 580-594. [CrossRef] [PubMed]

26. Lizundia, E.; Vilas, J.L.; León, L.M. Crystallization, structural relaxation and thermal degradation in Poly(L-lactide)/cellulose nanocrystal renewable nanocomposites. Carbohydr. Polym. 2015, 123, 256-265. [CrossRef] [PubMed]

27. Kim, I.H.; Jeong, Y.G. Polylactide/exfoliated graphite nanocomposites with enhanced thermal stability, mechanical modulus, and electrical conductivity. J. Polym. Sci. B 2010, 48, 850-858. [CrossRef]

28. Fukushima, K.; Murariu, M.; Camino, G.; Dubois, P. Effect of expanded graphite/layered-silicate clay on thermal, mechanical and fire retardant properties of poly(lactic acid). Polym. Degrad. Stab. 2010, 95, 1063-1076. [CrossRef]

29. Chen, Y.; Yao, X.; Zhou, X.; Pan, Z.; Gu, Q. Poly(lactic acid)/graphene nanocomposites prepared via solution blending using chloroform as a mutual solvent. J. Nanosci. Nanotechnol. 2011, 11, 7813-7819. [CrossRef] [PubMed]

30. Chieng, B.W.; Ibrahim, N.A.; Wan Yunus, W.M.Z. Optimization of Tensile Strength of Poly(Lactic Acid)/Graphene Nanocomposites Using Response Surface Methodology. Polym.-Plast. Technol. Eng. 2012, 51, 791-799. [CrossRef]

31. Mortazavi, B.; Hassouna, F.; Laachachi, A.; Rajabpour, A.; Ahzi, S.; Chapron, D.; Toniazzo, V.; Ruch, D. Experimental and multiscale modeling of thermal conductivity and elastic properties of PLA/expanded graphite polymer nanocomposites. Thermochim. Acta 2013, 552, 106-113. [CrossRef]

32. Li, X.; Xiao, Y.; Bergeret, A.; Longerey, M.; Che, J. Preparation of polylactide/graphene composites from liquid-phase exfoliated graphite sheets. Polym. Compos. 2014, 35, 396-403. [CrossRef]

33. Fu, Y.; Liu, L.; Zhang, J. Manipulating Dispersion and Distribution of Graphene in PLA through Novel Interface Engineering for Improved Conductive Properties. ACS Appl. Mater. Interfaces 2014, 6, 14069-14075. [CrossRef] [PubMed] 
34. Fu, Y.; Liu, L.; Zhang, J.; Hiscox, W.C. Functionalized graphenes with polymer toughener as novel interface modifier for property-tailored polylactic acid/graphene nanocomposites. Polymer 2014, 55, 6381-6389. [CrossRef]

35. Mittal, V.; Chaudhry, A.U.; Luckachan, G.E. Biopolymer-Thermally reduced graphene nanocomposites: Structural characterization and properties. Mater. Chem. Phys. 2014, 147, 319-332. [CrossRef]

36. Norazlina, H.; Kamal, Y. Graphene modifications in polylactic acid nanocomposites: A review. Polym. Bull. 2015, 72, 931-961. [CrossRef]

37. Ibarra-Gomez, R.; Muller, R.; Bouquey, M.; Rondin, J.; Serra, C.A.; Hassouna, F.; El Mouedden, Y.; Toniazzo, V.; Ruch, D. Processing of nanocomposites PLA/graphite using a novel elongational mixing device. Polym. Eng. Sci. 2015, 55, 214-222. [CrossRef]

38. Kashi, S.; Gupta, R.K.; Baum, T.; Kao, N.; Bhattacharya, S.; Sati, N. Morphology, electromagnetic properties and electromagnetic interference shielding performance of poly lactide/graphene nanoplatelet nanocomposites. Mater. Des. 2016, 95, 119-126. [CrossRef]

39. Liu, C.; Ye, S.; Feng, J. Promoting the dispersion of graphene and crystallization of poly(lactic acid) with a freezing-dried graphene/PEG masterbatch. Compos. Sci. Technol. 2017, 144, 215-222. [CrossRef]

40. Scaffaro, R.; Botta, L.; Maio, A.; Gallo, G. PLA graphene nanoplatelets nanocomposites: Physical properties and release kinetics of an antimicrobial agent. Compos. B 2017, 109, 138-146. [CrossRef]

41. Prashantha, K.; Roger, F. Multifunctional properties of 3D printed poly(lactic acid)/graphene nanocomposites by fused deposition modeling. J. Macromol. Sci. A 2017, 54, 24-29. [CrossRef]

42. Yu, W.W.; Zhang, J.; Wu, J.R.; Wang, X.Z.; Deng, Y.H. Incorporation of graphitic nano-filler and poly(lactic acid) in fused deposition modeling. J. Appl. Polym. Sci. 2017, 134, 44703. [CrossRef]

43. Mngomezulu, M.E.; Luyt, A.S.; Chapple, S.A.; John, M.J. Effect of expandable graphite on thermal and flammability properties of poly(lactic acid)-starch/poly(e-caprolactone) blend systems. Polym. Eng. Sci. 2017. [CrossRef]

44. Botlhoko, O.J.; Ramontja, J.; Ray, S.S. Thermal, mechanical, and rheological properties of graphite- and graphene oxide-filled biodegradable polylactide/poly(e-caprolactone) blend composites. J. Appl. Polym. Sci. 2017, 134. [CrossRef]

45. Wu, W.; Wu, C.-K.; Peng, H.; Sun, Q.; Zhou, L.; Zhuang, J.; Cao, X.; Roy, V.A.L.; Li, R.K.Y. Effect of nitrogen-doped graphene on morphology and properties of immiscible poly(butylene succinate)/polylactide blends. Compos. B. 2017, 113, 300-307. [CrossRef]

46. Goncalves, C.; Goncalves, I.C.; Magalhaes, F.D.; Pinto, A.M. Poly(lactic acid) composites containing carbon-based nanomaterials: A review. Polymers 2017, 9, 269. [CrossRef]

47. Yu, J.; Wang, N.; Ma, X. Fabrication and Characterization of Poly(lactic acid)/Acetyl Tributyl Citrate/Carbon Black as Conductive Polymer Composites. Biomacromolecules 2008, 9, 1050-1057. [CrossRef] [PubMed]

48. Wang, N.; Zhang, X.; Ma, X.; Fang, J. Influence of carbon black on the properties of plasticized poly(lactic acid) composites. Polym. Degrad. Stab. 2008, 93, 1044-1052. [CrossRef]

49. Wang, X.; Zhuang, Y.; Dong, L. Study of carbon black-filled poly(butylene succinate)/polylactide blend. J. Appl. Polym. Sci. 2012, 126, 1876-1884. [CrossRef]

50. Frackowiak, S.; Ludwiczak, J.; Leluk, K.; Orzechowski, K.; Kozlowski, M. Foamed poly(lactic acid) composites with carbonaceous fillers for electromagnetic shielding. Mater. Des. 2015, 65, 749-756. [CrossRef]

51. Lee, T.-W.; Jeong, Y.G. Enhanced electrical conductivity, mechanical modulus, and thermal stability of immiscible polylactide/polypropylene blends by the selective localization of multi-walled carbon nanotubes. Compos. Sci. Technol. 2014, 103, 78-84. [CrossRef]

52. Pluta, M.; Paul, M.-A.; Alexandre, M.; Dubois, P. Plasticized polylactide/clay nanocomposites. II. The effect of aging on structure and properties in relation to the filler content and the nature of its organo-modification. Polym. Sci. Polym. Phys. 2006, 44, 312-325. [CrossRef]

53. Zhou, Q.; Xanthos, M. Nanosize and microsize clay effects on the kinetics of the thermal degradation of polylactides. Polym. Degrad. Stab. 2009, 94, 327-338. [CrossRef]

54. Katiyar, V.; Gerds, N.; Koch, C.B.; Risbo, J.; Hansen, H.C.B.; Plackett, D. Melt processing of poly(L-lactic acid) in the presence of organomodified anionic or cationic clays. J. Appl. Polym. Sci. 2011, 122, 112-125. [CrossRef] 
55. Carrasco, F.; Gamez-Perez, J.; Santana, O.O.; Maspoch, M.L. Processing of poly(lactic acid)/organo-montmorillonite nanocomposites: Microstructure, thermal stability and kinetics of the thermal decomposition. Chem. Eng. J. 2011, 178, 451-460. [CrossRef]

56. Gerds, N.; Katiyar, V.; Koch, C.B.; Hansen, H.C.B.; Plackett, D.; Larsen, E.H.; Risbo, J. Degradation of 1-polylactide during melt processing with layered double hydroxides. Polym. Degrad. Stab. 2012, 97, 2002-2009. [CrossRef]

57. Androsch, R.; Di Lorenzo, M.L. Crystal nucleation in glassy poly(L-lactic acid). Macromolecules 2013, 46, 6048-6056. [CrossRef]

58. Jalali, A.; Huneault, M.A.; Elkoun, S. Effect of thermal history on nucleation and crystallization of poly(lactic acid). J. Mater. Sci. 2016, 51, 7768-7779. [CrossRef]

59. Volpe, V.; De Filitto, M.; Klofacova, V.; De Santis, F.; Pantani, R. Effect of mold opening on the properties of PLA samples obtained by foam injection molding. Polym. Eng. Sci. 2017. [CrossRef]

60. Volpe, V.; Pantani, R. Effect of processing condition on properties of polylactic acid parts obtained by foam injection molding. J. Cell. Plast. 2016, 53, 491-502. [CrossRef]

61. Mauro, M.; Cipolletti, V.; Galimberti, M.; Longo, P.; Guerra, G. Chemically Reduced Graphite Oxide with Improved Shape Anisotropy. J. Phys. Chem. C 2012, 116, 24809-24813. [CrossRef]

62. Yous, M.; Alix, S.; Lebeau, M.; Soulestin, J.; Lacrampe, M.F.; Krawczak, P. Evaluation of rheological properties of non-Newtonian fluids in micro rheology compounder: Experimental procedures for a reliable polymer melt viscosity measurement. Polym. Test. 2014, 40, 207-217.

63. De Santis, F.; Pantani, R. Melt compounding of poly(lactic Acid) and talc: Assessment of material behavior during processing and resulting crystallization. J. Polym. Res. 2015, 22, 242. [CrossRef]

64. Moad, G.; Dagley, I.J.; Habsuda, J.; Garvey, C.J.; Li, G.; Nichols, L.; Simon, G.P.; Nobile, M.R. Aqueous hydrogen peroxide-induced degradation of polyolefins: A greener process for controlled-rheology polypropylene. Polym. Degrad. Stab. 2015, 117, 97-108. [CrossRef]

65. Lin, Q.; Li, T.; Liu, Z.; Song, Y.; He, L.; Hu, Z.; Guo, Q.; Ye, H. High-resolution TEM observations of isolated rhombohedral crystallites in graphite blocks. Carbon 2012, 50, 2347-2374. [CrossRef]

66. Freise, E.J.; Kelly, A. The deformation of graphite crystals and the production of the rhombohedral form. Philos. Mag. 1963, 8, 1519-1533. [CrossRef]

67. Li, Z.Q.; Lu, C.J.; Xia, Z.P.; Zhou, Y.; Luo, Z. X-ray diffraction patterns of graphite and turbostratic carbon. Carbon 2007, 45, 1686-1695. [CrossRef]

68. Maggio, M.; Acocella, M.R.; Guerra, G. Intercalation compounds of oxidized carbon black. RSC Adv. 2016, 6, 105565-105572. [CrossRef]

69. Gorrasi, G.; Pantani, R. Hydrolysis and biodegradation of Poly(lactic acid). Adv. Polym. Sci. 2017, 1-33. [CrossRef]

70. Lopez-Ramon, M.V.; Stoeckli, F.; Moreno-Castilla, C.; Carrasco-Marın, F. Specific and non-specific interactions of water molecules with carbon surfaces from immersion calorimetry. Carbon 2000, 38, 825-829. [CrossRef] 